\title{
Role of DNA repair defects in predicting immunotherapy response
}

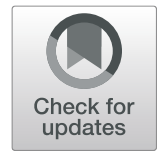

Jing Zhang, David J. H. Shih and Shiaw-Yih Lin ${ }^{*}$

\begin{abstract}
Defect in DNA damage response (DDR) is a common feature of cancer cells, which regulates tumor growth and therapeutic response. Recently, the approval of immune checkpoint blockade (ICB) for tumors with defective mismatch repair has paved the way for investigating the role of other DDR defects in sensitizing cancer to ICB therapy. Despite great progress in understanding DDR pathways, the mechanisms that link DDR defects and ICB response remain incompletely understood. Further, the clinical activity of ICB in patients with DDR defective tumors has not been well described. Here, we discuss recent studies demonstrating that biomarkers in DDR pathways may serve as potential predictors to guide the selection of patients for ICB therapy. A better understanding of the relationship between deficiency in DDR and response to ICB would facilitate efforts in optimizing the efficacy of immunotherapy.
\end{abstract}

Keywords: DNA damage response, Immunotherapy, Biomarkers, Immune checkpoint blockade

\section{Introduction}

Immune checkpoint blockade (ICB), using antibodies against inhibitory signaling molecules expressed on tumor and immune cells, has shown unprecedented clinical activity across many tumor types. Currently, the approved use of anti-CTLA-4 (ipilimumab), anti-PD-1 (nivolumab and pembrolizumab), anti-PD-L1 (atezolizumab, avelumab and durvalumab), and their combinations have demonstrated significant improvements over chemotherapy in cancer patients $[1,2]$. Despite this success, the majority of patients fail to respond to these ICB therapy due to the innate and acquired resistance [3].

Optimal design of ICB treatments will require multiple reliable predictive biomarkers that can help to select, before the initiation of treatment, patients who are most likely to benefit. For instance, Hodi et al. led a phase 3 ipilimumab study on patients with metastatic melanoma. They found that $20 \%$ of patients with metastatic melanoma achieved long-term benefit when treated with ipilimumab, $60 \%$ of patients had response for less than 2 years, and the rest

\footnotetext{
* Correspondence: sylin@mdanderson.org

Department of Systems Biology, The University of Texas MD Anderson Cancer Center, Houston, TX 77030, USA
}

showed no clinical benefit [4]. If we could predict, before ipilimumab treatment, whether a patient will be a longterm responder, short-term responder, or non-responder, we would be able to achieve the best therapeutic outcomes for the responders without causing unnecessary harm to non-responders. In a separate trial, Hodi et al. reported that $64 \%$ of patients treated with the nivolumab plus ipilimumab combination were still alive after 2 years [5]. However, this improvement in overall survival with the combination treatment came at significant cost: $54 \%$ of patients in the combination arm showed grade 3-4 adverse events, which is significantly higher than the ipilimumabonly arm (20\%). Indeed, biomarkers would be critical to guide the selection of patients for increasingly efficacious yet also increasingly toxic ICB treatments. Currently, there are few biomarkers that effectively predict tumor response to ICB, so the search continues for such biomarkers. A promising area to conduct this search is at the interplay between cancer defects in DNA repair and the anti-cancer immune response.

In order to preserve the integrity of their genomes, cells initiate DNA damage response (DDR) pathways for different types of DNA damage. Based on the DNA

C C The Author(s). 2020 Open Access This article is licensed under a Creative Commons Attribution 4.0 International License, which permits use, sharing, adaptation, distribution and reproduction in any medium or format, as long as you give appropriate credit to the original author(s) and the source, provide a link to the Creative Commons licence, and indicate if changes were made. The images or other third party material in this article are included in the article's Creative Commons. licence, unless indicated otherwise in a credit line to the material. If material is not included in the article's Creative Commons licence and your intended use is not permitted by statutory regulation or exceeds the permitted use, you will need to obtain permission directly from the copyright holder. To view a copy of this licence, visit http://creativecommons.org/licenses/by/4.0/. The Creative Commons Public Domain Dedication waiver (http://creativecommons.org/publicdomain/zero/1.0/) applies to the data made available in this article, unless otherwise stated in a credit line to the data. 
lesion, DDR comprises 5 major pathways, including mismatch repair (MMR), homology-dependent recombination (HR), non-homologous end joining (NHEJ), base excision repair (BER), and nucleotide excision repair (NER) [6] (Fig. 1). Defects in DDR pathways result in a variety of genomic aberrations and accelerate tumor development [7]. However, in addition to promoting tumorigenesis, DDR defects in many cancers also provide therapeutic opportunities to kill cancer cells without affecting normal cells. An successful example of targeting DDR defects is the use of poly(ADP ribose) polymerase (PARP) inhibitors for the treatment of HR defective cancers [8-11]. When the activity of PARP is inhibited, normal cells survive owing to their ability to repair damaged DNA via the HR pathway. In contrast, cancer cells with HR defect caused, for example, by mutations in BRCA1/2, BRD4, PTEN or other HR related genes are sensitive to PARP inhibitors [12, 13]. Mounting evidence indicates that DDR defects are also important in driving sensitivity and response to ICB. To date, microsatellite instability/defective mismatch repair (MSI/dMMR) is a validated DDR defect biomarker for predicting response to ICB therapy that is approved by FDA [14]. The potential for defects in other DDR pathways to serve as predictive biomarkers for ICB response is less well investigated. In this review, we summarize the emerging evidence that elucidates the relationship between DDR pathways and ICB response, and we also discuss the promising roles of DNA repair proteins as predictive biomarkers to guide the use of ICB therapy.

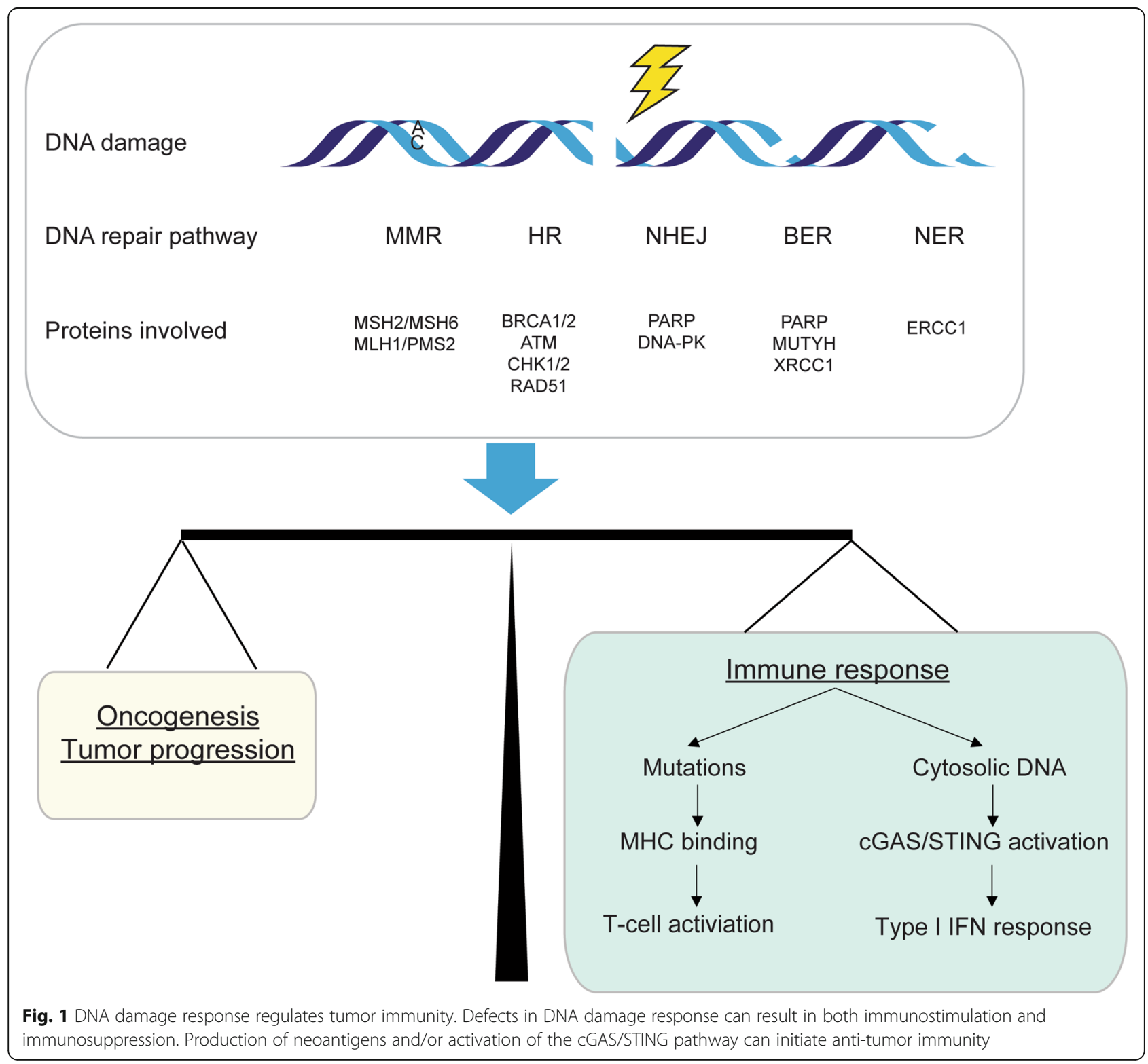




\section{Existing predictive biomarkers for ICB in solid tumors}

\section{PD-L1 as a predictive biomarker}

Due to the importance of PD-L1 pathway in cancer development, PD-L1 (also known as CD274 or B7-H1) expression is one of the earliest and most promising predictive biomarkers. The initial phase 1 study in 2012 assessed the antitumor activity of nivolumab in patients with various advanced solid tumors [15]. Their study suggested that PD-L1 expression in tumors may predict clinical outcomes to anti-PD-1 therapy across many tumor types. Similar findings were reported by Taube et al. in 2014 who found that the pre-treatment level of PD-L1 expression is correlated with response to anti-PD-1 blockade [16]. Interestingly, PD-L1 expression by immune cells but not by tumor cells can also predicts the response to ICB across multiple cancer types $[17,18]$.

However, evidence from other studies shows that a substantial portion $(20-30 \%)$ of PD-L1 negative patients respond to anti-PD-1 therapy $[19,20]$, which weakens the use of only PD-L1 status for predicting response. In addition, multiple other studies did not find that PD-L1 positive patients benefit from anti-PD-1 therapy [21, 22], which could be explained by the complexity of PD-L1 biology. PD-L1 expression is inducible and changes dynamically $[23,24]$, and PD-L1 is stored in intracellular reserves: tumor cells may translocate intracellular PD-L1 to the cell surface following the clearance of anti-PD-L1 antibodies [25]. Additionally, there is no standardized criteria and cutoff threshold for assessing PD-L1 expression level, which compromises the accurate evaluation of PD-L1 status $[26,27]$. These studies collectively highlight important limitations of using PD-L1 as a sole predictive biomarker for ICB treatment.

\section{Tumor mutation burden and other potential biomarkers}

Tumor mutation burden (TMB) is another promising predictive biomarker of ICB response. It measures the total number of tumor mutations, often within limited genomic regions, using high-throughput sequencing technologies [28]. The association of TMB with anti-PD-L1 or antiCTLA4 therapy has been widely investigated. A clinical study in 2014 found that melanoma patients with higher pre-therapy TMB derived durable clinical benefit from anti-CTLA-4 treatment, though the authors noted that TMB alone was not sufficient to predict response accurately [29]. Indeed, this association between TMB and ICB was confirmed by another study of patients with melanoma [30]. By analyzing the sequencing data from pretherapy tumors, the authors discovered that TMB and neoantigen load were significantly associated with clinical benefit from ICB. Similar findings linking TMB and ICB were observed in many other tumor types, including non- small-cell lung cancer (NSCLC) [21,31], small cell lung cancer (SCLC) [32], and urothelial carcinoma [33, 34].

Despite the significant correlation between TMB and anti-PD-L1 therapy in various tumor types [28], many patients with high TMB do not respond to ICB and vice versa. For instance, patients with renal cell carcinoma [35], squamous cell carcinoma of the head and neck [36], breast or pancreatic cancer [37] show no significant association between TMB and response to ICB. Interestingly, a bioinformatic analysis of 68 patients with melanoma concluded that changes in TMB after 4 weeks of nivolumab treatment was strongly associated with antiPD-1 response [38]. While this finding may be useful in early assessment of patient's response to ICB, it would be challenging to implement it into clinical practice due to the requirement of on-therapy biopsies. In addition, the assays for measuring TMB, such as whole exome sequencing and targeted next generation sequencing [39], are expensive and complex. Clinical applications of these assays would require further standardization of these assays, including the determination of the optimal cutpoint for distinguishing tumors with high vs. low TMB.

With the improved understanding of tumor immune microenvironment, several promising candidates have emerged as predictive biomarkers. For example, the density of tumor-infiltrating lymphocytes (TILs) within the tumor may predict response to anti-PD1 therapy [40, 41]. Furthermore, STK11/LKB1 mutations in lung adenocarcinoma was correlated with primary resistance to anti-PD1 therapy [42]. Interestingly, in a study of 35 patients with metastatic clear cell renal cell carcinoma, patients who have PBRM1 loss can benefit from antiPD-L1 therapy [43], and the authors validated this association in a follow-up study [44]. However, McDermott et al. reported that PBRM1 mutations were not associated with anti-PD-L1 therapy based on the results from a phase 2 study in 305 patients with renal cell carcinoma [35]. The author instead showed that that blocking VEGF may sensitize patients to ICB treatment. Therefore, the biology of PBRM1 and ICB response is likely complex and remains largely unexplored. In patients with melanoma or uterine leiomyosarcoma, loss of PTEN is associated with inferior outcomes with antiPD1 therapy [45, 46]. Additionally, recent studies suggested that EGFR or ALK mutant patients with NSCLC do not respond to pembrolizumab irrespective of PD-L1 expression [47-49].

Above all, there is a growing number of studies identifying predictive biomarkers for ICB response. These studies collectively suggest that, owing to the complexity of tumor-immune dynamics, single biomarkers are insufficient to accurately predict clinical outcomes with $\mathrm{ICB}$, and optimal prediction of ICB response will require the identification of additional predictor biomarkers. Due to 
the interaction between DDR pathways and immune response, studying how DDR pathways influence antitumor immunity may provide fruitful insights, as well as revealing biomarkers of DDR deficiency that predict clinical response to ICB.

\section{The association between DDR defects and immunotherapy response $\mathrm{MSI} / \mathrm{dMMR}$}

To date, the strongest evidence for the relationship between DDR defects and response to ICB therapy involves the DNA mismatch repair pathway. MSI together with dMMR are two broadly used predictive biomarkers [50]. MMR pathway is an essential DNA repair mechanism that identifies and repairs mismatched bases during DNA replication and genetic recombination [51]. dMMR, due to mutations or promoter methylation of any of four key genes-MLH1, MSH2, MSH6 and PMS2-leads to the accumulation of mismatch errors and results in MSI and tumorigenesis [52]. Although MSI or dMMR rarely appears in breast cancer [53], prostate cancer [54] and lung adenocarcinoma [55], they are widely observed in other tumor types, including colorectal cancer [56, 57], gastric and gastrointestinal cancer $[58,59]$, and endometrial cancer [60].

Given that dMMR tumors harbor a large number of mutations, which are associated with high neoantigen load and T-cell infiltration [61, 62], it is not surprising that dMMR tumors can respond well to immune checkpoint blockade. A phase 2 clinical trial in 2015 investigated the activity of pembrolizumab in 41 patients who had either MMR-deficient or MMR-proficient metastatic carcinomas [63]. This study showed that patients with MMR-deficient tumors had higher response rates to anti-PD-L1 therapy than patient with MMR-proficient tumors. In an expanded study across 12 different tumor types, the authors found that around $20 \%$ of patients had achieved complete response, confirming that dMMR is a useful biomarker for predicting the response to ICB [64]. Based on these findings, the FDA, for the first time in its history, approved the use of pembrolizumab for the treatment of tumors that are indicated solely by biomarkers, irrespective of tumor site [65].

Although MSI/dMMR can serve as effective biomarkers to predict ICB response, some tumors with MSI high display resistance to ICB treatment, which challenges the idea that MSI/dMMR can be used as the sole biomarker in all tumor types $[66,67]$. Therefore, identifying other DDR related biomarkers would be a promising avenue for predicting clinical response to ICB treatment.

\section{HR defects}

Among these widely studied DNA repair pathways, HR is an accurate and error-free pathway for repairing double strand breaks. Accumulating evidence points to the potential association between HR defect and ICB response. In high grade serous ovarian cancer, mutations in $B R C A 1 / B R C A 2$, which are essential players in $\mathrm{HR}$ pathways, result in higher neoantigen load, increased TILs, as well as enhanced expressions of PD-1 and PDL1 [68]. Similar findings were observed in patients with pancreatic ductal adenocarcinoma [69]. Elevated TILs have also been observed in BRCA1/BRCA2 mutant prostate cancer [70, 71] and breast cancer [72, 73]. In contrast, a pooled analysis of five phase 2 studies showed that TIL density was not associated with HR defect or $B R C A 1 / 2$ mutation in early stage patients with triple negative breast cancer [74].

In addition, the use of ICB therapy in HR deficient cancers has showed conflictive results. By analyzing the whole exome sequences of 38 patients with melanoma previously treated with $\mathrm{PD}-1$ inhibitors, Hugo et al. reported that ICB responders are enriched for $B R C A 2 \mathrm{mu}-$ tations [75]. Further, a patient with $B R C A 2$ mutant MSS prostate cancer was reported to be sensitive to ICB [71]. A phase 1b study of patients with recurrent or refractory ovarian cancer demonstrated that BRCA status was not associated with response to anti-PD-L1 treatment [76]. Together, these results indicate that whether HR defect increase susceptibility to ICB treatment remains to be determined.

\section{BER deficiency}

DNA single strand breaks, which do not disrupt the helical structure of DNA, are detected and repaired through BER pathway [77]. BER defective tumors from patients with colon adenocarcinoma, breast invasive carcinoma, or uterine corpus endometrial carcinoma, exhibit increased neoantigen production and upregulated PD-L1 expression [78]. Prominent TIL has been observed in colorectal cancer patients with germline mutations in $M U T Y H$, a key DNA glycosylase involved in BER [79, 80]. These studies hint that BER defective cancer may display increased susceptibility to ICB. However, clinical trials regarding the use of ICB in BER defective cancer have not been reported.

\section{NER defects}

Bulky DNA adducts that significantly distort the DNA helix, caused by UV irradiation or alkylating agents, are repaired by NER pathway [81]. NSCLC patients with single nucleotide polymorphisms in the ERCC1 gene (which encodes the XPF nuclease that incises the damaged segment of the DNA during NER) were more sensitive to anti-PD-L1 treatment [82]. In fact, the role of NER in sensitizing ICB therapy in other tumor types is less well characterized. Further studies should be performed to investigate whether biomarkers of NER defects could predict clinical benefit from ICB. 


\section{POLD1/ POLE mutations}

Additional potential markers of genomic instability and response to immunotherapy are mutations in polymerase delta 1 (POLD1) and polymerase epsilon (POLE). These proteins play important role in DDR and are essential for accurate DNA replication by proofreading. Similar to the phenotype of dMMR, POLD1 or POLE mutant tumors displayed increased TMB, neoantigen load, TILs, and effector cytokine levels [83-86], indicating that these mutated tumors may respond to ICB therapy. Recently, Wang et al. analyzed POLE/POLD1 mutations in 47,721 patients with different types of cancer and found that POLE/POLD1 mutations are promising predictive biomarkers for ICB [87]. Ongoing ICB trials are enrolling patients with $P O L E$-mutant tumors [88].

The relationships between DDR defects and ICB response are summarized in Table 1.

\section{Mechanisms underlying the effect of DDR deficiency in antitumor immunity}

In the tumor microenvironment, defects in DDR can dramatically impact the balance between immune surviellence and tumor progression. Failed DNA damage repair results in the accumulation of genomic errors, which activates oncogenes and initiates tumor development $[89,90]$. On the other hand, this genomic instability may trigger anti-tumor immune response through two major mechanisms (Fig. 1). The accumulation of mutations caused by defective DDR can encode tumorspecific neoantigens that are presented on the cell surface in the context of major histocompatibility complex class I. These neoantigens are, in turn, specifically recognized by $T$ cells [91] and enhance the anti-tumor immune response. In support of the notion that DDR defects drive ICB response via the production of neoantigens, DDR mutations in gastroesophageal cancers are associated with high TMB and elevated PD-L1 expression [92]. In urothelial cancer, patients whose tumors harbored any mutations in DDR pathways showed better response to anti-PD-1/PD-L1 treatment [93].

However, numerous studies have showed that tumors with very few mutations can also be sensitive to ICB treatment [94]. Therefore, non-neoantigen-based mechanisms of DDR defects on ICB treatment have been proposed. Failure of DDR can result in the increase of cytosolic DNA, which binds to the cyclic GMP-AMP synthase (cGAS) and subsequently stimulates the innate immune response through STING pathway (Fig. 1) [9598]. In contrast to DDR intact tumors, DDR deficient breast tumors had increased immune infiltration and elevated PD-L1 expression, due to cGAS/STING pathway activation instead of neoantigen production [99]. In cellbased assays, loss of BRCA2 stimulated a cGAS/STING mediated interferon response [100]. Importantly, STING activation mediated by DNA-damaging agents is implicated in response to ICB therapy. Due to the important role of PARP inhibitors in inducing synthetic lethality in cancer cells with HR defect, multiple studies recently investigated the clinical activity of combination therapy with PARP inhibitors and anti PD-1/PD-L1 in different cancer types $[101,102]$. Although this combination therapy showed promising anti-tumor activity, tumors from the responders with ovarian carcinoma are BRCA1/2 wild-type or HR intact [103]. The observation that HR intact tumors may also respond to the PARP inhibitor and ICB combination could perhaps be explained by the the activation of the cGAS/STING [104]. Further, a preclinical study showed that STING-inducing CHK1

Table 1 Summary of the relationships between DDR defects and ICB response

\begin{tabular}{|c|c|c|c|c|}
\hline $\begin{array}{l}\text { DDR } \\
\text { defects }\end{array}$ & Tumor types & Criteria & Immunologic features & ICB response \\
\hline \multirow[t]{2}{*}{$\begin{array}{l}\text { MSI/ } \\
\text { dMMR }\end{array}$} & $\begin{array}{l}\text { Colon, } \\
\text { endometrial, } \\
\text { gastric, ovarian }\end{array}$ & $\begin{array}{l}\text { Germline mutations in DNA } \\
\text { mismatch repair genes: } M L H 1, M S H 2 \text {, } \\
\text { MSH6 or PMS2 }\end{array}$ & $\begin{array}{l}\text { Increased TMB, neoantigen load, } \\
\text { TILs, and expression of PD-1 or } \\
\text { PD-L1 }\end{array}$ & $\begin{array}{l}\text { Patients with MMR-deficient tumors had im- } \\
\text { proved response rate to anti-PD-1 therapy: } \\
\text { NCT01876511 }\end{array}$ \\
\hline & & & & $\begin{array}{l}\text { Ongoing trials: NCT02563002, NCT02912572, } \\
\text { NCT02899793, NCT03150706, NCT03435107 }\end{array}$ \\
\hline \multirow[t]{2}{*}{$\begin{array}{l}\text { HR } \\
\text { defects }\end{array}$} & $\begin{array}{l}\text { Ovarian, breast, } \\
\text { prostate, } \\
\text { pancreatic }\end{array}$ & $\begin{array}{l}\text { Germline mutations in BRCA1 or } \\
B R C A 2\end{array}$ & $\begin{array}{l}\text { Increased neoantigen load, TILs, } \\
\text { and expression of PD-1 or PD- } \\
\text { L1 }\end{array}$ & $\begin{array}{l}\text { ICB therapy in HR deficient cancers has } \\
\text { shown conflicting results: NCT01772004, Refs. } \\
{[71,75]}\end{array}$ \\
\hline & & & & $\begin{array}{l}\text { Ongoing trials: NCT03025035, NCT03428802, } \\
\text { NCT02571725, NCT03101280, NCT02849496 }\end{array}$ \\
\hline $\begin{array}{l}\text { POLD1/ } \\
\text { POLE } \\
\text { mutations }\end{array}$ & $\begin{array}{l}\text { Endometrial, } \\
\text { colon, }\end{array}$ & $\begin{array}{l}\text { Germline mutations in POLD1 or } \\
\text { POLE }\end{array}$ & $\begin{array}{l}\text { Increased TMB, neoantigen load, } \\
\text { TILs, and effector cytokine levels }\end{array}$ & $\begin{array}{l}\text { Ongoing trials: NCT02912572, NCT02899793, } \\
\text { NCT03150706, NCT03435107, NCT03428802 }\end{array}$ \\
\hline $\begin{array}{l}\text { BER } \\
\text { deficiency }\end{array}$ & $\begin{array}{l}\text { Colon, breast, } \\
\text { endometrial }\end{array}$ & Germline mutations in MUTYH & $\begin{array}{l}\text { Increased neoantigen load and } \\
\text { PD-L1 expression }\end{array}$ & Not reported \\
\hline $\begin{array}{l}\text { NER } \\
\text { defects }\end{array}$ & Lung & $\begin{array}{l}\text { Single nucleotide polymorphisms in } \\
\text { ERCC1 }\end{array}$ & & Not reported \\
\hline
\end{tabular}


inhibitors may enhance efficacy of ICB treatment in patients with SCLC [105].

In addition to neoantigen production and cGAS/ STING pathway, DDR deficiency may also increase tumor sensitivity by activating other signaling pathways. For example, inhibition of ATM has been identified to induce interferon-mediated innate immune response in a cGAS/STING independent, but TBK1- and SRCdependent manner [106]. Furthermore, blocking Nedd8mediated clearance pathway in an MSI and ICB-resistant syngeneic mouse model has been shown to improve the efficacy of anti-PD1 treatment [67]. Despite the extensive knowledge of DDR pathways, it remains largely unclear how DDR defects initiate anti-tumor immunity to enhance response to ICB.

\section{Conclusions and future directions}

Although ICB induces robust anti-tumor immune responses across different cancer types, it remains challenging to identify reliable predictive biomarkers that guide patient selection for ICB treatment. Currently, only a few biomarkers are FDA-approved for clinical use, including PD-L1 expression and MSI/dMMR [1, 14]. Other emerging biomarker candidates, such as TMB, the density of TILs, and immunogenic neoantigen load, have shown their unique advantages as well as their own intrinsic limitations. Based on the current understanding of the clinical response to ICB, a single biomarker is not sufficient to predict who will likely benefit from ICB treatment.

In addition to the widely studied MSI/dMMR, defects in other major DDR pathways have shown to promote PD-L1 expression, elevate TILs, and increase neoantigen load, which are potential determinants of the response to ICB. Interestingly, patients with co-mutations in multiple DDR pathways exhibit favorable clinical response to ICB [107]. Therefore, it is important to better understand how biomarkers in various DDR pathways can serve as effective predictors of ICB response. Prospective pre-clinical and clinical studies are needed to fully understand the relationship between DDR defects and ICB response.

Because cancer cells are often defective in DDR and poorly tolerate further DNA damage, combination treatments of ICB immunotherapy with DNA damaging therapeutics may enhance therapy response. In fact, several ongoing trials are evaluating the efficacy of combining ICB therapy and PARP inhibitors [108]. Other inhibitors of DDR pathways, such as inhibitors of ATM, ATR, CHK1, CHK2, may also contribute to ICB combination. DDR deficiency plays a critical role in immunotherapy response, future research in this area would likely provide insightful clues for the finding the optimal multimodality combination therapy, as well as for discovering additional biomarkers that can collectively predict immunotherapy response and maximize clinical benefit.

\section{Abbreviations}

DDR: DNA damage response; ICB: Immune checkpoint blockade;

MMR: Mismatch repair; HR: Homology-dependent recombination; NHEJ: Nonhomologous end joining; BER: Base excision repair; NER: Nucleotide excision repair; PARP: Poly(ADP ribose) polymerase; MSI: Microsatellite instability; dMMR: Defective mismatch repair; TMB: Tumor mutation burden; NSCLC: Non-small-cell lung cancer; SCLC: Small cell lung cancer; TILs: Tumorinfiltrating lymphocytes

\section{Acknowledgements \\ Not applicable.}

\section{Authors' contributions}

JZ wrote and edited the manuscript. DJHS edited the manuscript. SYL contributed to conception and critical revision of the manuscript. All authors read and approved the final manuscript.

Funding

This work was supported by a grant from the NCI (R01CA218287) to SYL.

Availability of data and materials

Not applicable.

Ethics approval and consent to participate

Not applicable.

Consent for publication

Not applicable.

Competing interests

The authors declare that they have no competing interests.

Received: 21 April 2020 Accepted: 22 June 2020

Published online: 29 June 2020

References

1. Wei SC, Duffy CR, Allison JP. Fundamental mechanisms of immune checkpoint blockade therapy. Cancer Discov. 2018;8(9):1069-86.

2. Akinleye A, Rasool Z. Immune checkpoint inhibitors of PD-L1 as cancer therapeutics. J Hematol Oncol. 2019;12(1):92.

3. Jenkins RW, Barbie DA, Flaherty KT. Mechanisms of resistance to immune checkpoint inhibitors. Br J Cancer. 2018;118(1):9-16.

4. Hodi FS, et al. Improved survival with ipilimumab in patients with metastatic melanoma. N Engl J Med. 2010;363(8):711-23.

5. Hodi FS, et al. Combined nivolumab and ipilimumab versus ipilimumab alone in patients with advanced melanoma: 2-year overall survival outcomes in a multicentre, randomised, controlled, phase 2 trial. Lancet Oncol. 2016:17(11):1558-68.

6. Lord CJ, Ashworth A. The DNA damage response and cancer therapy. Nature. 2012:481(7381):287-94.

7. Burrell RA, et al. The causes and consequences of genetic heterogeneity in cancer evolution. Nature. 2013;501(7467):338-45.

8. Farmer $\mathrm{H}$, et al. Targeting the DNA repair defect in BRCA mutant cells as a therapeutic strategy. Nature. 2005;434(7035):917-21.

9. Bryant $\mathrm{HE}$, et al. Specific killing of BRCA2-deficient tumours with inhibitors of poly(ADP-ribose) polymerase. Nature. 2005;434(7035):913-7.

10. Michèle R, et al. PARP inhibition: PARP1 and beyond. Nat Rev Cancer. 2010; 10(4):293.

11. Matulonis UA, et al. Olaparib maintenance therapy in patients with platinum-sensitive, relapsed serous ovarian cancer and a BRCA mutation: overall survival adjusted for postprogression poly(adenosine diphosphate ribose) polymerase inhibitor therapy. Cancer. 2016;122(12):1844-52.

12. Sun C, et al. BRD4 inhibition is synthetic lethal with PARP inhibitors through the induction of homologous recombination deficiency. Cancer Cell. 2018; 33(3):401-416.e8.

13. Javle M, Curtin NJ. The role of PARP in DNA repair and its therapeutic exploitation. Br J Cancer. 2011;105(8):1114-22. 
14. Lemery S, Keegan P, Pazdur R. First FDA approval agnostic of cancer site when a biomarker defines the indication. N Engl J Med. 2017;377(15):1409-12.

15. Topalian SL, et al. Safety, activity, and immune correlates of anti-PD-1 antibody in cancer. N Engl J Med. 2012;366(26):2443-54.

16. Taube JM, et al. Association of PD-1, PD-1 ligands, and other features of the tumor immune microenvironment with response to anti-PD-1 therapy. Clin Cancer Res. 2014;20(19):5064-74.

17. Herbst RS, et al. Predictive correlates of response to the anti-PD-L1 antibody MPDL3280A in cancer patients. Nature. 2014;515(7528):563-7.

18. Mariathasan $\mathrm{S}$, et al. TGF $\beta$ attenuates tumour response to PD-L1 blockade by contributing to exclusion of T cells. Nature. 2018;554(7693):544-8.

19. Weber JS, et al. Nivolumab versus chemotherapy in patients with advanced melanoma who progressed after anti-CTLA-4 treatment (CheckMate 037): a randomised, controlled, open-label, phase 3 trial. Lancet Oncol. 2015;16(4): 375-84.

20. Robert C, et al. Nivolumab in previously untreated melanoma without BRAF mutation. N Engl J Med. 2015;372(4):320-30.

21. Carbone DP, et al. First-line nivolumab in stage IV or recurrent non-smallcell lung cancer. N Engl J Med. 2017;376(25):2415-26.

22. Bellmunt J, et al. Pembrolizumab as second-line therapy for advanced urothelial carcinoma. N Engl J Med. 2017;376(11):1015-26.

23. Garcia-Diaz A, et al. Interferon receptor signaling pathways regulating PD-L1 and PD-L2 expression. Cell Rep. 2017;19(6):1189-201.

24. Thiem A, et al. IFN-gamma-induced PD-L1 expression in melanoma depends on p53 expression. J Exp Clin Cancer Res. 2019;38(1):397.

25. Wu Y, et al. PD-L1 distribution and perspective for cancer immunotherapyblockade, knockdown, or inhibition. Front Immunol. 2019;10:2022.

26. Hirsch FR, et al. PD-L1 immunohistochemistry assays for lung cancer: results from phase 1 of the blueprint PD-L1 IHC assay comparison project. J Thorac Oncol. 2017;12(2):208-22.

27. McLaughlin J, et al. Quantitative assessment of the heterogeneity of PD-L1 expression in non-small-cell lung cancer. JAMA Oncol. 2016;2(1):46-54.

28. Yarchoan M, Hopkins A, Jaffee EM. Tumor mutational burden and response rate to PD-1 inhibition. N Engl J Med. 2017;377(25):2500-1.

29. Snyder A, et al. Genetic basis for clinical response to CTLA-4 blockade in melanoma. N Engl J Med. 2014;371(23):2189-99.

30. Van Allen EM, et al. Genomic correlates of response to CTLA-4 blockade in metastatic melanoma. Science. 2015;350(6257):207-11.

31. Hellmann MD, et al. Nivolumab plus ipilimumab in lung cancer with a high tumor mutational burden. N Engl J Med. 2018;378(22):2093-104

32. Hellmann MD, et al. Tumor mutational burden and efficacy of nivolumab monotherapy and in combination with ipilimumab in small-cell lung cancer. Cancer Cell. 2018;33(5):853-861.e4.

33. Rosenberg JE, et al. Atezolizumab in patients with locally advanced and metastatic urothelial carcinoma who have progressed following treatment with platinum-based chemotherapy: a single-arm, multicentre, phase 2 trial. Lancet. 2016:387(10031):1909-20.

34. Kao C, et al. Tumor mutational burden (TMB) as a predictive biomarker of immune checkpoint blockade (ICB) in metastatic solid tumors. J Clin Oncol. 2020;38(5_suppl):80.

35. McDermott DF, et al. Clinical activity and molecular correlates of response to atezolizumab alone or in combination with bevacizumab versus sunitinib in renal cell carcinoma. Nat Med. 2018;24(6):749-57.

36. Hanna GJ, et al. Frameshift events predict anti-PD-1/L1 response in head and neck cancer. JCI Insight. 2018;3(4):e98811.

37. McGrail DJ, et al. Multi-omics analysis reveals neoantigen-independent immune cell infiltration in copy-number driven cancers. Nat Commun. 2018; 9(1):1317.

38. Riaz N, et al. Tumor and microenvironment evolution during immunotherapy with nivolumab. Cell. 2017;171(4):934-949.e16.

39. Endris $V$, et al. Measurement of tumor mutational burden (TMB) in routine molecular diagnostics: in silico and real-life analysis of three larger gene panels. Int J Cancer. 2019;144(9):2303-12.

40. Tumeh PC, et al. PD-1 blockade induces responses by inhibiting adaptive immune resistance. Nature. 2014;515(7528):568-71.

41. Galon J, et al. Type, density, and location of immune cells within human colorectal tumors predict clinical outcome. Science. 2006;313(5795):1960-4.

42. Skoulidis F, et al. STK11/LKB1 mutations and PD-1 inhibitor resistance in KRAS-mutant lung adenocarcinoma. Cancer Discov. 2018;8(7):822-35.

43. Miao D, et al. Genomic correlates of response to immune checkpoint therapies in clear cell renal cell carcinoma. Science. 2018;359(6377):801-6.
44. Braun DA, et al. Clinical validation of PBRM1 alterations as a marker of immune checkpoint inhibitor response in renal cell carcinoma. JAMA Oncol. 2019;5(11):1631-3.

45. Peng $W$, et al. Loss of PTEN promotes resistance to $T$ cell-mediated immunotherapy. Cancer Discov. 2016;6(2):202-16.

46. George $\mathrm{S}$, et al. Loss of PTEN is associated with resistance to anti-PD-1 checkpoint blockade therapy in metastatic uterine leiomyosarcoma. Immunity. 2017;46(2):197-204.

47. Gandhi $L$, et al. Pembrolizumab plus chemotherapy in metastatic nonsmall-cell lung cancer. N Engl J Med. 2018;378(22):2078-92.

48. Gainor JF, et al. EGFR mutations and ALK rearrangements are associated with low response rates to PD-1 pathway blockade in non-small cell lung cancer: a retrospective analysis. Clin Cancer Res. 2016;22(18):4585-93.

49. Lisberg A, et al. A phase II study of pembrolizumab in EGFR-mutant, PD-L1+ tyrosine kinase inhibitor naive patients with advanced NSCLC. J Thorac Oncol. 2018;13(8):1138-45

50. Zhao P, et al. Mismatch repair deficiency/microsatellite instability-high as a predictor for anti-PD-1/PD-L1 immunotherapy efficacy. J Hematol Oncol. 2019;12(1):54.

51. Iyer RR, et al. DNA mismatch repair: functions and mechanisms. Chem Rev. 2006;106(2):302-23.

52. Lynch HT, et al. Making sense of missense in Lynch syndrome: the clinical perspective. Cancer Prev Res. 2010;3(11):1371-4.

53. Mills AM, et al. The relationship between mismatch repair deficiency and PD-L1 expression in breast carcinoma. Am J Surg Pathol. 2018;42(2):183-91.

54. Ryan S, Jenkins MA, Win AK. Risk of prostate cancer in Lynch syndrome: a systematic review and meta-analysis. Cancer Epidemiol Biomark Prev. 2014; 23(3):437-49.

55. Takamochi K, et al. DNA mismatch repair deficiency in surgically resected lung adenocarcinoma: microsatellite instability analysis using the Promega panel. Lung Cancer. 2017;110:26-31.

56. Pino MS, et al. Deficient DNA mismatch repair is common in Lynch syndrome-associated colorectal adenomas. J Mol Diagn. 2009;11(3):238-47.

57. Thibodeau SN, Bren G, Schaid D. Microsatellite instability in cancer of the proximal colon. Science. 1993;260(5109):816-9.

58. Yamamoto $\mathrm{H}$, Imai K, Perucho M. Gastrointestinal cancer of the microsatellite mutator phenotype pathway. J Gastroenterol. 2002;37(3):153-63.

59. Yamamoto $\mathrm{H}$, et al. Gastric cancers of the microsatellite mutator phenotype display characteristic genetic and clinical features. Gastroenterology. 1999; 116(6):1348-57.

60. Kato $M$, et al. DNA mismatch repair-related protein loss as a prognostic factor in endometrial cancers. J Gynecol Oncol. 2015;26(1):40-5.

61. Llosa NJ, et al. The vigorous immune microenvironment of microsatellite instable colon cancer is balanced by multiple counter-inhibitory checkpoints. Cancer Discov. 2015;5(1):43-51.

62. Schwitalle $Y$, et al. Immune response against frameshift-induced neopeptides in HNPCC patients and healthy HNPCC mutation carriers. Gastroenterology. 2008;134(4):988-97.

63. Le DT, et al. PD-1 blockade in tumors with mismatch-repair deficiency. N Engl J Med. 2015;372(26):2509-20.

64. Le DT, et al. Mismatch repair deficiency predicts response of solid tumors to PD-1 blockade. Science. 2017;357(6349):409-13.

65. Prasad V, Kaestner V, Mailankody S. Cancer drugs approved based on biomarkers and not tumor type-FDA approval of pembrolizumab for mismatch repair-deficient solid cancers. JAMA Oncol. 2018;4(2):157-8.

66. Yang RK, et al. Identification of biomarkers of immune checkpoint blockade efficacy in recurrent or refractory solid tumor malignancies. Oncotarget. 2020;11(6):600-18.

67. McGrail DJ, et al. Proteome instability is a therapeutic vulnerability in mismatch repair-deficient cancer. Cancer Cell. 2020;37(3):371-386.e12.

68. Strickland KC, et al. Association and prognostic significance of BRCA1/2mutation status with neoantigen load, number of tumor-infiltrating lymphocytes and expression of PD-1/PD-L1 in high grade serous ovarian cancer. Oncotarget. 2016:7(12):13587-98.

69. Connor AA, et al. Association of distinct mutational signatures with correlates of increased immune activity in pancreatic ductal adenocarcinoma. JAMA Oncol. 2017;3(6):774-83.

70. Jenzer $\mathrm{M}$, et al. The BRCA2 mutation status shapes the immune phenotype of prostate cancer. Cancer Immunol Immunother. 2019;68(10):1621-33.

71. Panda $A$ et al. Role for immune checkpoint blockade in BRCA2-mutant prostate cancer. J Clin Oncol. 2019;37(8_suppl):59. 
72. Green AR, et al. Clinical impact of tumor DNA repair expression and T-cell infiltration in breast cancers. Cancer Immunol Res. 2017;5(4):292-9.

73. Pellegrino $B$, et al. Homologous recombination repair deficiency and the immune response in breast cancer: a literature review. Transl Oncol. 2020; 13(2):410-22.

74. Telli ML, et al. Association of tumor infiltrating lymphocytes with homologous recombination deficiency and BRCA1/2 status in patients with early triple-negative breast cancer: a pooled analysis. Clin Cancer Res. 2019: clincanres.0664.2019. https://clincancerres.aacrjournals.org/content/early/201 9/12/03/1078-0432.CCR-19-0664.

75. Hugo W, et al. Genomic and transcriptomic features of response to anti-PD1 therapy in metastatic melanoma. Cell. 2016;165(1):35-44.

76. Disis ML, et al. Efficacy and safety of avelumab for patients with recurrent or refractory ovarian cancer: phase $1 \mathrm{~b}$ results from the JAVELIN solid tumor trial. JAMA Oncol. 2019;5(3):393-401.

77. David SS, O'Shea VL, Kundu S. Base-excision repair of oxidative DNA damage. Nature. 2007:447(7147):941-50.

78. Permata TBM, et al. Base excision repair regulates PD-L1 expression in cancer cells. Oncogene. 2019;38(23):4452-66.

79. Colebatch $\mathrm{A}$, et al. The role of MYH and microsatellite instability in the development of sporadic colorectal cancer. Br J Cancer. 2006;95(9):1239-43.

80. Nielsen M, et al. Colorectal carcinomas in MUTYH-associated polyposis display histopathological similarities to microsatellite unstable carcinomas. BMC Cancer. 2009;9:184.

81. Cleaver JE, Lam ET, Revet I. Disorders of nucleotide excision repair: the genetic and molecular basis of heterogeneity. Nat Rev Genet. 2009;10(11): 756-68.

82. Aiello MM, et al. Excision repair cross complementation group 1 (ERCC-1) gene polymorphisms and response to nivolumab in advanced non-small cell lung cancer (NSCLC). J Clin Oncol. 2017;35(15_suppl):3032.

83. van Gool IC, et al. POLE proofreading mutations elicit an antitumor immune response in endometrial cancer. Clin Cancer Res. 2015;21(14):3347-55.

84. Howitt BE, et al. Association of polymerase e-mutated and microsatelliteinstable endometrial cancers with neoantigen load, number of tumorinfiltrating lymphocytes, and expression of PD-1 and PD-L1. JAMA Oncol. 2015;1(9):1319-23.

85. Mehnert JM, et al. Immune activation and response to pembrolizumab in POLE-mutant endometrial cancer. J Clin Invest. 2016;126(6):2334-40.

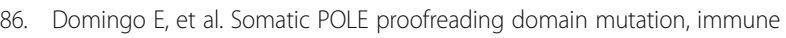
response, and prognosis in colorectal cancer: a retrospective, pooled biomarker study. Lancet Gastroenterol Hepatol. 2016;1(3):207-16.

87. Wang $F$, et al. Evaluation of POLE and POLD1 mutations as biomarkers for immunotherapy outcomes across multiple cancer types. JAMA Oncol. 2019; 5(10):1504-6.

88. Mouw KW, et al. DNA damage and repair biomarkers of immunotherapy response. Cancer Discov. 2017;7(7):675-93.

89. Duijf $\mathrm{PHG}$, et al. Mechanisms of genomic instability in breast cancer. Trends Mol Med. 2019;25(7):595-611.

90. McGrail DJ, et al. Defective replication stress response is inherently linked to the cancer stem cell phenotype. Cell Rep. 2018;23(7):2095-106.

91. Yarchoan $\mathrm{M}$, et al. Targeting neoantigens to augment antitumour immunity Nat Rev Cancer. 2017;17(4):209-22.

92. Cerniglia M, et al. Association of DNA damage response and repair genes (DDR) mutations and microsatellite instability (MSI), PD-L1 expression, tumor mutational burden (TMB) in gastroesophageal cancers. J Clin Oncol. 2019; 37(4_suppl):60.

93. Teo MY, et al. Alterations in DNA damage response and repair genes as potential marker of clinical benefit from PD-1/PD-L1 blockade in advanced urothelial cancers. J Clin Oncol. 2018:36(17):1685-94.

94. Chabanon RM, et al. Mutational landscape and sensitivity to immune checkpoint blockers. Clin Cancer Res. 2016;22(17):4309-21.

95. Li T, Chen ZJ. The CGAS-CGAMP-STING pathway connects DNA damage to inflammation, senescence, and cancer. J Exp Med. 2018;215(5):1287-99.

96. Chen Q, Sun L, Chen ZJ. Regulation and function of the cGAS-STING pathway of cytosolic DNA sensing. Nat Immunol. 2016:17(10):1142-9.

97. Motwani M, Pesiridis S, Fitzgerald KA. DNA sensing by the CGAS-STING pathway in health and disease. Nat Rev Genet. 2019;20(11):657-74.

98. Kwon J, Bakhoum SF. The cytosolic DNA-sensing CGAS-STING pathway in cancer. Cancer Discov. 2020;10(1):26-39.
99. Parkes EE, et al. Activation of STING-dependent innate immune signaling by S-phase-specific DNA damage in breast cancer. J Natl Cancer Inst. 2016; 109(1):djw199.

100. Heijink AM, et al. BRCA2 deficiency instigates CGAS-mediated inflammatory signaling and confers sensitivity to tumor necrosis factor-alpha-mediated cytotoxicity. Nat Commun. 2019;10(1):100.

101. Lee J-M, et al. Safety and clinical activity of the programmed death-ligand 1 inhibitor durvalumab in combination with poly (ADP-ribose) polymerase inhibitor Olaparib or vascular endothelial growth factor receptor 1-3 inhibitor Cediranib in women's cancers: a dose-escalation, phase I study. J Clin Oncol. 2017;35(19):2193-202.

102. Stewart RA, Pilie PG, Yap TA. Development of PARP and immunecheckpoint inhibitor combinations. Cancer Res. 2018;78(24):6717-25.

103. Konstantinopoulos PA, et al. Single-arm phases 1 and 2 trial of Niraparib in combination with pembrolizumab in patients with recurrent platinumresistant ovarian carcinoma. JAMA Oncol. 2019;5(8):1141-9.

104. Shen J, et al. PARPi triggers the STING-dependent immune response and enhances the therapeutic efficacy of immune checkpoint blockade independent of BRCAness. Cancer Res. 2019;79(2):311-9.

105. Sen T, et al. Targeting DNA damage response promotes antitumor immunity through STING-mediated T-cell activation in small cell lung cancer. Cancer Discov. 2019:9(5):646-61.

106. Zhang Q, et al. Inhibition of ATM increases interferon signaling and sensitizes pancreatic cancer to immune checkpoint blockade therapy. Cancer Res. 2019;79(15):3940-51.

107. Wang Z, et al. Comutations in DNA damage response pathways serve as potential biomarkers for immune checkpoint blockade. Cancer Res. 2018; 78(22):6486-96.

108. Li A, et al. Prospects for combining immune checkpoint blockade with PARP inhibition. J Hematol Oncol. 2019;12(1):98.

\section{Publisher's Note}

Springer Nature remains neutral with regard to jurisdictional claims in published maps and institutional affiliations.

Ready to submit your research? Choose BMC and benefit from:

- fast, convenient online submission

- thorough peer review by experienced researchers in your field

- rapid publication on acceptance

- support for research data, including large and complex data types

- gold Open Access which fosters wider collaboration and increased citations

- maximum visibility for your research: over $100 \mathrm{M}$ website views per year

At $\mathrm{BMC}$, research is always in progress.

Learn more biomedcentral.com/submissions 